\title{
Sea ice is a mushy layer
}

\author{
Article
}

Published Version

Feltham, D. L., Untersteiner, N., Wettlaufer, J. S. and Worster, M. G. (2006) Sea ice is a mushy layer. Geophysical Research Letters, 33 (14). L14501. ISSN 0094-8276 doi: https://doi.org/10.1029/2006GL026290 Available at https://centaur.reading.ac.uk/34911/

It is advisable to refer to the publisher's version if you intend to cite from the work. See Guidance on citing.

Published version at: http://dx.doi.org/10.1029/2006GL026290

To link to this article DOI: http://dx.doi.org/10.1029/2006GL026290

Publisher: American Geophysical Union

All outputs in CentAUR are protected by Intellectual Property Rights law, including copyright law. Copyright and IPR is retained by the creators or other copyright holders. Terms and conditions for use of this material are defined in the End User Agreement.

\section{$\underline{\text { www.reading.ac.uk/centaur }}$}

\section{CentAUR}

Central Archive at the University of Reading

Reading's research outputs online 


\title{
Sea ice is a mushy layer
}

\author{
D. L. Feltham, ${ }^{1,2}$ N. Untersteiner, ${ }^{3}$ J. S. Wettlaufer, ${ }^{4}$ and M. G. Worster ${ }^{5}$ \\ Received 13 March 2006; revised 31 May 2006; accepted 6 June 2006; published 19 July 2006.
}

[1] Sea ice is a two-phase, two-component, reactive porous medium: an example of what is known in other contexts as a mushy layer. The fundamental conservation laws underlying the mathematical description of mushy layers provide a robust foundation for the prediction of seaice evolution. Here we show that the general equations describing mushy layers reduce to the model of Maykut and Untersteiner (1971) under the same approximations employed therein. Citation: Feltham, D. L., N. Untersteiner, J. S. Wettlaufer, and M. G. Worster (2006), Sea ice is a mushy layer, Geophys. Res. Lett., 33, L14501, doi:10.1029/ 2006GL026290.

\section{Introduction}

[2] The large-scale heat and mass balance of the sea-ice cover has been extensively studied because of its importance in climate. The principal balances that control the distribution of sea-ice thickness are those between thermal processes, that lead to growth and melting, and mechanical processes, induced by wind, oceanic and internal stresses, that drive deformation [Thorndike, 1992, and references therein]. In numerical simulations of these processes the most common approach is to treat the thermal evolution one-dimensionally and couple this to sea-ice motion and deformation.

[3] Treatments of the heat conduction through sea ice vary in complexity, ranging from thermodynamically based approaches with an empirically constrained vertical salinity profile [Untersteiner, 1964; Maykut and Untersteiner, 1971] to those with linear temperature gradients (the 'zero-layer' Semtner model [Semtner, 1976]), used in many global circulation studies. Still others may treat, for example, melt ponds and lead fraction [Ebert and Curry, 1993] and yet prescribe a uniform salinity. From the perspective of the physical realism underlying the heat conduction process in geophysical parameter regimes, most models subsequent to that of Maykut and Untersteiner [1971] are either simplifications or approximations that are often inconsistent with the governing conservation laws of mass and energy. Here, we show that the Untersteiner [1964] and hence Maykut and Untersteiner [1971] treatments are natural consequen-

\footnotetext{
${ }^{1}$ Centre for Polar Observation and Modelling, Department of Earth Sciences, University College London, London, UK.

${ }^{2}$ British Antarctic Survey, Cambridge, UK.

${ }^{3}$ Department of Atmospheric Sciences, University of Washington, Seattle, Washington, USA.

${ }^{4}$ Department of Geology and Geophysics and Department of Physics, Yale University, New Haven, Connecticut, USA.

${ }^{5}$ Institute of Theoretical Geophysics, Department of Applied Mathematics and Theoretical Physics, Cambridge University, Cambridge, UK.

Copyright 2006 by the American Geophysical Union. 0094-8276/06/2006GL026290
}

ces of a basic treatment of the conservation laws governing the thermodynamics and dynamics of two-phase, twocomponent systems known as mushy layers. Simplifications of these approaches should satisfy the same fundamental conservation laws, and their predictions can be validated in the context of small-scale process studies against those of the full mushy-layer theory.

\section{Theory of Mushy Layers}

[4] A mushy layer consists of a rigid matrix of pure solid (ice) bathed in its impurity rich melt (brine). The conservation laws describing the evolution of mushy layers quantitatively explain experiments on laboratory-grown sea ice [Wettlaufer et al., 1997a, 1997b] and field measurements [Wettlaufer et al., 2000]. Theoretical studies of the mushy layer equations specifically applied to sea ice have been performed by Feltham and Worster [1999], Feltham et al. [2002], Neufeld et al. [2006], and Taylor and Feltham [2004]. For our purposes the term "mushy-layer" is equivalent to "sea ice" but we use this nomenclature, more broadly employed in continuum mechanics and materials science, to emphasize the inviolability of conservation laws and the utility of the concepts shown to be quantitatively accurate. The equations describing conservation of heat and composition for a region containing elements of solid phase and its melt are reviewed by Worster [1992, 2000]. Here we show how this theory applies to sea ice. The local energy balance in a small region within the sea ice, containing both brine and ice, is given by

$$
c_{m} \frac{\partial T}{\partial t}+c_{b} \mathbf{u} \cdot \nabla T=\nabla \cdot\left(k_{m} \nabla T\right)+\mathcal{L} \frac{\partial \phi}{\partial t}+A_{R},
$$

where $T$ is temperature expressed in degrees Kelvin and $\phi$ is the solid volume fraction. In this equation, $c_{m}=c_{i} \phi+c_{b}(1-\phi)$ is the effective volumetric specific heat capacity of the sea ice and is related to the volumetric specific heat capacity of the solid ice $\left(c_{i}\right)$ and brine $\left(c_{b}\right)$. The velocity $\mathbf{u}$ is defined as the volume flux of brine per unit perpendicular cross-section area and is known as the Darcy velocity. For a random distribution of solid and liquid phases, Batchelor [1974] showed that $k_{m} \leq k_{i} \phi+k_{b}(1-\phi)$ where $k_{i}$ and $k_{b}$ are the thermal conductivities of the solid (ice) and liquid (brine) phases respectively. The inequality reduces to an equality in the case that the solid and liquid layers are aligned in planes parallel to the heat flux. In sea ice, the solid phase takes the form of platelets, aligned vertically and hence we can use $k_{m}=k_{i} \phi+k_{b}(1-\phi)$ to a very good approximation. The symbol $\mathcal{L}=\rho_{i}\left(H_{b}-H_{i}\right)$, a latent heat of fusion per unit volume, is strictly the local difference between the enthalpies of brine $H_{b}$ and ice $H_{i}$ multiplied by the density of ice $\rho_{i}$. In general it is a function 
of both temperature and composition. But if local equilibrium is assumed (see equation (3) below) then it is solely a function of temperature. The final term in the energy balance $A_{R}$ represents the absorption of shortwave radiation that has passed through the upper sea-ice surface. The particular model of absorption chosen is not the topic of this paper but we note that Beer's law, describing radiation absorption in a semi-infinite, non-scattering medium has been widely used [e.g., Maykut and Untersteiner, 1971; Ebert and Curry, 1993]. A more sophisticated two-stream radiation model has also been used in thermodynamic simulations of thin sea ice, Taylor and Feltham [2004].

[5] Local conservation of salt is given by

$$
(1-\phi) \frac{\partial C}{\partial t}+\mathbf{u} \cdot \nabla C=\nabla \cdot\left(D_{m} \nabla C\right)+\frac{\rho_{i}}{\rho_{b}}\left(C-C_{i}\right) \frac{\partial \phi}{\partial t},
$$

where $C$ is the concentration of salt in the brine, $C_{i}$ is the concentration of salt incorporated into the ice crystals (and is nearly zero), and $\rho_{b}$ is the brine density. To an excellent approximation, $D_{m}=(1-\phi) D_{b}$, where $D_{m}$ and $\left(D_{b}\right)$ are the diffusivities of salt in the mushy layer and brine. The final term in equation (2) is responsible for brine expulsion, given that $\rho_{i}<\rho_{b}$. However, detailed solutions of the mushy layer equations show that while this redistributes salt a little it does not result in any transport of salt to the ocean [Notz, 2006]. It is important to note that both the theory of mushy layers and the thermodynamic model of sea ice of Maykut and Untersteiner [1971] make use of an assumption that sea ice is in local thermodynamic equilibrium. This assumption is justified provided that the rate of phase change within the sea ice is sufficiently rapid so that thermodynamic equilibrium can be maintained between the solid and liquid elements. This is satisfied if the timescale of interstitial solute transport, $\delta^{2} / D_{b}$, where $\delta$ is the solid-element spacing in the sea ice, is short compared to the timescale of overall temperature variations within the sea ice. Since the heating rate of the sea ice scales with elapsed time $t$, thermodynamic equilibrium is maintained once $t>\delta^{2} / D_{b}$. For sea ice, $\delta \approx$ $10^{-4}-10^{-3} \mathrm{~m}$ and $D_{b} \approx 10^{-9} \mathrm{~m}^{2} \mathrm{~s}^{-1}$ so that thermodynamic equilibrium is maintained after approximately $10 \mathrm{~s}$ to $10^{3} \mathrm{~s}$. This time interval is short compared to the timescale of diurnal variation within a sea-ice layer on the polar oceans. The assumption of local thermodynamic equilibrium implies that the temperature and brine concentration lie on the liquidus curve in the equilibrium phase diagram for sea ice. This curve can be approximated over the temperature (and pressure) range typical of polar ocean surface water by a straight line so that

$$
T_{L}(C)=T_{L}(0)-\Gamma C,
$$

where $\Gamma$ is a positive constant. This equation links the equilibrium freezing or liquidus temperature $T_{L}$ of brine to its salt concentration; for example, if $C=0$, then $T_{L}(C=0)=273 \mathrm{~K}$, the freezing point of pure water. We note that over the temperature range experienced within the bulk of the sea ice the nonlinearity of the liquidus relationship becomes important for quantitative accuracy [Notz, 2006]. However, this effect does not change the core principles we are dealing with here. The assumption of local thermo- dynamic equilibrium, $T=T_{L}(C)$, can be used to eliminate brine salinity, $C$, from equation (2), yielding

$$
(1-\phi) \frac{\partial T}{\partial t}+\mathbf{u} \cdot \nabla T=\nabla \cdot\left(D_{m} \nabla T\right)+\frac{\rho_{i}}{\rho_{b}}\left[T-T_{L}\left(C_{i}\right)\right] \frac{\partial \phi}{\partial t} .
$$

[6] Equations (1)-(3), together with an equation describing the dynamics of interstitial fluid flow (e.g., Darcy's equation) form the fundamental mathematical description of a mushy layer. There are now several experimental confirmations of their predictions [e.g., Worster, 1986; Shirtcliffe et al., 1991; Wettlaufer et al., 1997a; Aussillous et al., 2006]. Their validity is thus well established and they can be applied with confidence to the study of sea ice.

\section{Equivalence of Mushy Layer Theory and Existing Thermodynamic Sea Ice Models}

[7] Interpretations and explanations vary, but it is a fact that nearly all of the essential observational facts of sea-ice formation and its physical and chemical properties, among others, were described by Malmgren [1927]. He was the first to consider the effects of trapped seawater on the thermophysical properties of sea ice, for example.

[8] We now show that the conservation equations of energy and salt are equivalent, under imposition of the same physical assumptions, as existing sea ice models that are based upon the model of Maykut and Untersteiner [1971]. In particular, we set $\rho_{i}=\rho_{b}$ and $D_{m}=0$ in equation (4). This eliminates the processes of so-called "brine expulsion" and "brine-pocket diffusion" [Untersteiner, 1968]. We introduce the bulk salinity

$$
C_{\text {bulk }}(z, t)=\phi C_{i}+(1-\phi) C
$$

which is the local concentration of salt per unit volume of sea ice (ice and brine); it is the concentration of the brine that would be obtained if a local volume of sea ice were completely melted. From (5), we see that

$$
\phi=\frac{C_{b u l k}-C}{C_{i}-C}=\frac{T_{L}\left(C_{b u l k}\right)-T}{T_{L}\left(C_{i}\right)-T},
$$

where the second equation follows from the first using the liquidus relationship. Substituting for the solid fraction in terms of $C_{\text {bulk }}$ and $T$, equations (1) and (2) may, respectively, be re-written as

$$
\begin{aligned}
& \left\{c_{m}+\mathcal{L} \frac{T_{L}\left(C_{i}\right)-T_{L}\left(C_{\text {bulk }}\right)}{\left(T_{L}\left(C_{i}\right)-T\right)^{2}}\right\} \frac{\partial T}{\partial t} \\
& \quad+\left\{c_{b}+\frac{\mathcal{L}}{T_{L}\left(C_{i}\right)-T}\right\} \mathbf{u} \cdot \nabla T=\nabla \cdot\left(k_{m} \nabla T\right)+A_{R},
\end{aligned}
$$

and

$$
\frac{\partial C_{b u l k}}{\partial t} \approx \frac{1}{\Gamma}(\mathbf{u} \cdot \nabla T)
$$

The bulk salinity of perennial sea ice depends upon its history because processes acting throughout the layer affect its distribution [Untersteiner, 1968]. In most sea-ice models 
the bulk salinity is treated as uniform [Ebert and Curry, 1993] or dependent on ice thickness [e.g., Cox and Weeks, 1988] or is given by a prescribed, empirically based, timeindependent vertical profile [Maykut and Untersteiner, 1971]. We note that, across all types of ice, $\mathbf{u}$ is controlled by brine drainage and flushing, whereas for perennial ice flushing is dominant and that, while the theory of mushy layers can incorporate these effects in principle, calculating overall brine drainage is complex and these topics are the subject of current research.

[9] We see from equation (7) that there is an effective specific heat capacity $c_{\text {eff }}$ given by

$$
c_{\mathrm{eff}} \equiv \mathcal{L} \frac{T_{L}\left(C_{i}\right)-T_{L}\left(C_{b u l k}\right)}{\left(T_{L}\left(C_{i}\right)-T\right)^{2}}+\frac{T_{L}\left(C_{i}\right)-T_{L}\left(C_{b u l k}\right)}{\left(T_{L}\left(C_{i}\right)-T\right)}\left(c_{b}-c_{i}\right)+c_{i},
$$

where we have substituted for $c_{m}$ and $\phi$. Similarly, we can define the effective conductivity

$$
k_{\mathrm{eff}} \equiv k_{i}-\frac{T_{L}\left(C_{i}\right)-T_{L}\left(C_{b u l k}\right)}{\left(T_{L}\left(C_{i}\right)-T\right)}\left(k_{i}-k_{b}\right) .
$$

In order to compare our mushy layer model directly with that of Maykut and Untersteiner [1971], we must make the same assumptions made therein, namely we neglect brine flow $\mathbf{u}=\mathbf{0}$, consider the bulk salinity to be independent of time, and consider variation in the vertical direction only. In consequence our model reduces to

$$
c_{\text {eff }} \frac{\partial T}{\partial t}=\frac{\partial}{\partial z}\left(k_{\text {eff }} \frac{\partial T}{\partial z}\right)+A_{R} .
$$

This model is now of identical form to that of Maykut and Untersteiner [1971], with the phase change terms held implicitly within the effective heat capacity and conductivity. To demonstrate the equivalence, all that is now necessary is to show that our expressions for heat capacity and conductivity are of the same form, namely, the approximate forms derived by Untersteiner [1961]. Because these were found to be in agreement with both Schwerdtfeger's [1963] and Ono's [1967] theories for the range of temperatures and salinities observed in perennial sea ice, they were used by Maykut and Untersteiner [1971]. Schwerdtfeger obtained the effective sea-ice heat capacity per unit mass to be

$$
\frac{c_{s i}}{\rho_{s i}}=\frac{\Gamma C_{\text {bulk }}}{\theta^{2}} \frac{\mathcal{L}}{\rho_{i}}-\frac{\Gamma C_{\text {bulk }}}{\theta}\left(\frac{c_{w}}{\rho_{w}}-\frac{c_{i}}{\rho_{i}}\right)+\frac{c_{i}}{\rho_{i}}
$$

(his equation 1.3 .7 with $\mathcal{L} / \rho_{i}$ constant) and the conductivity of sea ice to be

$$
k_{s i}=k_{b i}+\left(k_{b i}-k_{b}\right) \frac{\rho_{s i}}{\rho_{w}} \frac{\Gamma C_{b u l k}}{\theta},
$$

(his equation 2.6.3), where quantities previously introduced are expressed in the notation of this paper. In these expressions, $\theta=T-273, c_{w}$ is the volumetric heat capacity of pure water, $c_{i}$ is the volumetric heat capacity of pure ice, $k_{b i}$ is the conductivity of bubbly ice, $k_{b}$ is the conductivity of brine, $\rho_{s i}$ is the sea-ice bulk density and $\rho_{w}$ is the density of water. The expressions for the effective volumetric heat capacity and thermal conductivity of sea ice that we have obtained can be simplified by using the following approximations: $c_{b}=c_{w}, k_{i}=k_{b i}, T-T_{L}\left(C_{i}\right)=\theta$, and $\rho_{i}=$ $\rho_{w}=\rho_{s i}$. In this case, we obtain

$$
c_{\text {eff }} \approx \frac{\Gamma C_{\text {bulk }}}{\theta^{2}} \mathcal{L}-\frac{\Gamma C_{\text {bulk }}}{\theta}\left(c_{i}-c_{w}\right)+c_{i},
$$

and

$$
k_{\mathrm{eff}} \approx k_{i}+\left(k_{b i}-k_{b}\right) \frac{\Gamma C_{b u l k}}{\theta} .
$$

Clearly, with these approximations, the mushy layer model for volumetric specific heat capacity and conductivity possess the form determined by Schwerdtfeger [1963] and Ono [1967] and hence used by Maykut and Untersteiner [1971], with the only small differences being (i) the volumetric versus per unit mass units and (ii) the absence of a density ratio of order unity in the mushy layer expression for conductivity.

\section{Conclusion}

[10] We have provided a simple demonstration of the equivalence of the mushy layer model of sea ice and the thermodynamic sea ice model of Maykut and Untersteiner [1971], and therefore Untersteiner [1964]. The importance of this demonstration has three principal components: Firstly, it is conceptually important that the thermodynamics of sea ice must obey the basic conservation laws provided by the theory of mushy layers. Secondly, the form of the equations exhibits the independent, but coupled role played by salt through a layer of sea ice. Namely, while the temperature and salinity of the interstitial brine within sea ice are coupled by the liquidus constraint, the total amount of salt within the sea ice at a given depth and the temperature are independent thermodynamic variables determining its state. Processes such as drainage and flushing link these independent variables. Thirdly, the fact that the theory has a firm physical foundation that has been quantitatively tested in the laboratory provides us with an inviolable set of constraints for both local process studies and generalizations or simplifications for climatological studies. Indeed, there is evidence for some confusion in the literature [e.g., Vancoppenolle et al., 2005; Tartinville et al., 2001], concerning the thermodynamic requirements for the conservation of mass and energy and what constitutes a prediction: for example, despite the acknowledgment that temperature and salinity are thermodynamically coupled variables, model runs of Vancoppenolle et al. [2005] are made with specified sea ice salinity properties that are neither empirically based nor constrained by observation. Furthermore, Tartinville et al. [2001] treat sea ice as a "negative reservoir" for salt; when ice is formed, salt is released into the ocean and when ice melts, salt is "extracted" from the ocean. Finally, we note that the theory of mushy layers provides the rubric for the development of appropriately simplified mean-field theories for possible incorporation into large scale models. 
[11] Acknowledgments. We thank D. Notz for comments on an earlier version of this paper. JSW thanks the Bosack and Kruger Foundation, the National Science Foundation, Yale University, Trinity College and the Department of Applied Mathematics and Theoretical Physics, University of Cambridge, for support during the sabbatical leave where this paper was written.

\section{References}

Aussillous, P., A. J. Sederman, L. F. Gladden, H. E. Huppert, and M. G. Worster (2006), Magnetic resonance imaging of structure and convection in solidifying mushy layers, J. Fluid Mech., 552, 99-125.

Batchelor, G. K. (1974), Transport properties of two-phase materials with random structure, Annu. Rev. Fluid Mech., 6, 227-255.

Cox, G. F. N., and W. F. Weeks (1988), Numerical simulations of the profile properties of undeformed first-year sea ice during the growth season, J. Geophys. Res., 93, 12,449-12,460.

Ebert, E. E., and J. A. Curry (1993), An intermediate one-dimensional thermodynamic sea-ice model for investigating ice-atmosphere interactions, J. Geophys. Res., 98, 10,085-10,109.

Feltham, D. L., and M. G. Worster (1999), Flow-induced morphological instability of a mushy layer, J. Fluid Mech., 391, 337-357.

Feltham, D. L., M. G. Worster, and J. S. Wettlaufer (2002), The influence of ocean flow on newly forming sea ice, J. Geophys. Res., 107(C2), 3009, doi:10.1029/2000JC000559.

Malmgren, F. (1927), On the properties of sea ice, in The Norwegian Polar Expedition 'Maud,' 1918-1925: Scientific Results, vol. 1, pp. 1-67, Geofys. Inst., Bergen, Norway.

Maykut, G. A., and N. Untersteiner (1971), Some results from a timedependent, thermodynamic model of sea ice, J. Geophys. Res., 76, $1550-1575$

Neufeld, J. A., J. S. Wettlaufer, D. L. Feltham, and M. G. Worster (2006), Corrigendum: Flow-induced morphological instability of a mushy layer, J. Fluid Mech., 549, 442-443.

Notz, D. (2006), Thermodynamic and fluid-dynamical processes in sea ice, Ph.D. thesis, Trinity College, Univ. of Cambridge, Cambridge, U. K.

Ono, N. (1967), Specific heat and heat of fusion of sea ice, in Physics of Snow and Ice, vol. 1, edited by H. Oura, pp. 599-610, Inst. of Low Temp. Sci., Hokkaido, Japan.

Schwerdtfeger, P. (1963), The thermal properties of sea ice, J. Glaciol., 4, 789-807.

Semtner, A. J. (1976), A model for the thermodynamic growth of sea ice in numerical investigations of climate, J. Phys. Oceanogr., 6, 379-389.

Shirtcliffe, T. G. L., H. E. Huppert, and M. G. Worster (1991), Measurement of the solid fraction in the crystallization of a binary melt, J. Crystal Growth, 113, 566-574.
Tartinville, B., J.-M. Campin, T. Fichefet, and H. Goosse (2001), Realistic representation of the surface freshwater flux in an ice-ocean general circulation model, Ocean Modell., 3, 95-108.

Taylor, P. D., and D. L. Feltham (2004), A model of melt pond evolution on sea ice, J. Geophys. Res., 109, C12007, doi:10.1029/2004JC002361.

Thorndike, A. S. (1992), Estimates of the sea ice thickness distribution using observations and theory, J. Geophys. Res., 97, 12,601-12,605.

Untersteiner, N. (1961), On the mass and heat budget of Arctic sea ice, Arch. Meteorol. Geophys. Bioklimatol., Ser. A, 12, 151-182.

Untersteiner, N. (1964), Calculations of temperature regime and heat budget of sea ice in the central Arctic, J. Geophys. Res., 69, 4755-4766.

Untersteiner, N. (1968), Natural desalination and equilibrium salinity profile of perennial sea ice, J. Geophys. Res., 73, 1251-1257.

Vancoppenolle, M., T. Fichefet, and C. M. Bitz (2005), On the sensitivity of undeformed Arctic sea ice to its vertical salinity profile, Geophys. Res. Lett., 32, L16502, doi:10.1029/2005GL023427.

Wettlaufer, J. S., M. G. Worster, and H. E. Huppert (1997a), The phase evolution of young sea ice, Geophys. Res. Lett., 24, 1251-1254.

Wettlaufer, J. S., M. G. Worster, and H. E. Huppert (1997b), Natural convection during solidification of an alloy from above with application to the evolution of sea ice, J. Fluid Mech., 344, 291-316.

Wettlaufer, J. S., M. G. Worster, and H. E. Huppert (2000), The solidification of leads: Theory, experiment and field observations, J. Geophys. Res., 105, 1123-1134.

Worster, M. G. (1986), Solidification of an alloy from a cooled boundary, J. Fluid Mech., 167, 481-501.

Worster, M. G. (1992), The dynamics of mushy layers, in Interactive Dynamics of Convection and Solidification, edited by S. H. Davis et al., pp. 113-138, Springer, New York.

Worster, M. G. (2000), Solidification of fluids, in Perspectives in Fluid Dynamics, edited by G. K. Batchelor, H. K. Moffatt, and M. G. Worster, pp. 393-446, Cambridge Univ. Press, New York.

D. L. Feltham, Centre for Polar Observation and Modelling, Department of Earth Sciences, University College London, Pearson Building, Gower Street, London WC1E 6BT, UK.

N. Untersteiner, Department of Atmospheric Sciences, University of Washington, Box 351640, Seattle, WA 98195-1640, USA.

J. S. Wettlaufer, Department of Geology and Geophysics and Department of Physics, Box 208109, Yale University, New Haven, CT 06520, USA. (john.wettlaufer@yale.edu)

M. G. Worster, Institute of Theoretical Geophysics, Department of Applied Mathematics and Theoretical Physics, Wilberforce Road, Cambridge University, Cambridge CB3 0WA, UK. 